\title{
Gait Analysis
}

\author{
M.Venkateswara Rao ${ }^{1}$, Dr.M.Malini ${ }^{1}$, O. Nageshwaramma ${ }^{1}$, B.Sai Lalitha ${ }^{1}$
}

\begin{abstract}
Gait analysis is the quantitative measurement and assessment of human locomotion. Gait is a cyclic activity and repeatable and certain discrete events have been defined as significant and need to be studied at depth to discriminate pathologic gait from normal gait. It can also be further extended to evaluate the rehabilitative devices and also guide the orthopedic surgeon for surgical intervention. Different research groups are working to quantify the gait parameters using different techniques. In this paper, a portable, wearable and easy to implement gait analysis system is proposed. The gait analysis data acquisition is composed of two triaxial accelerometers, a set of eight switches embedded in the sole of the shoes and a microcontroller. The data acquired from these sensors is then transferred to a PC, where data analysis is performed using MATLAB. The subjects were asked to wear the gadget and walk along a straight path of 400 meters at normal speed. The developed software acquires the data and analyze the same to acquire the important features of the gait. The data is collected from 25 control subjects, whose age is $22 \pm 2$ years and the gait parameters are determined.
\end{abstract}

Keywords - Gait, Wearable sensors, 3-Axis accelerometer.

\section{INTRODUCTION}

Gait analysis is a very important procedure in assessing and improving many quality of life indicators. For example, gait analysis can be used to improve the sport persons performance and injury prevention. It can also be mainly used for patients with diabetes, where gait analysis provides information about chances for development of foot ulceration and ways to prevent. For elderly persons and patients with peripheral neuropathy gait analysis provides valuable information. In certain neurological disorders also, gait pattern changes and a study of it provides information about the severity of the disease and can be used even for prognosis.

In the earlier days, the gait was video recorded for future comparison and/or observed by an expert and the problems were identified. This type of analysis is highly subjective and the comparison of past record was highly complicated/impossible and also error prone due to its subjective nature. The present standard method for human gait analysis is the optical motion analysis using high speed cameras to record human motion. To improve the performance of the system, active or passive markers are attached at predefined locations on the body. The integration of threedimension motion measurement using multi-camera system and reaction force measurement using force plates has been successfully used to tracking human body parts and performing dynamics analysis of their gait [1], [2]. However,

\footnotetext{
${ }^{1}$ Department of Biomedical Engineering, Osmania University, Hyderabad, India.
}

these methods are confined to laboratory environment and also require considerable lab space and high speed graphical signal processing methods, which increases the complexity and cost of the system.

Many researchers used various combinations of sensors including force sensitive resistors, inclinometers, gyroscopes, goniometers and accelerometers [3], [4], [5]. In addition to their competitive price, user friendliness, miniaturized for portability, capability of efficiently recording and processing large number of parameters in less time and space are among the required traits of such devices [6]. In order to achieve this goal a technique using MEMS based accelerometers and insole mounted switches is presented in this paper.

\section{METHODOLOGY}

The proposed system is built around a PIC 16F877 Microcontroller, with two tri-axial accelerometers, 8 membrane switches, X-bee modules as shown in Fig. 1. The MEMS based accelerometer from Free scale semiconductor, is considered as the sensor and it provides a digital output $\left(\mathrm{I}^{2} \mathrm{C} / \mathrm{SPI}\right)$ and is a low power, low profile capacitive micro machined accelerometer featuring signal conditioning, a low pass filter, temperature compensation, self-test and pulse detect for quick motion detection . $0 \mathrm{~g}$ offset and sensitivity are factory set and require no external devices. Two accelerometers are considered and both are interfaced to PIC 16F877 microcontroller through two separate $\mathrm{I}^{2} \mathrm{C}$ interfaces. Each 3-axis accelerometer is mounted on a shoe so that both the leg signals can be obtained. The accelerometers are attached to the shoes using special velcro tapes to avoid vibrational disturbances. (Fig. 2)

Each shoe is also provided with four membrane switches attached at the bottom side of the shoe at pre-defined locations. The locations considered are on the heel part, on the middle lateral side, on the metatarsal area and the last on the big toe. These positions are very important in defining the different phases of gait such as: initial contact, loading response, mid-stance, terminal stance, pre-swing, initial swing, mid-swing and terminal swing. The switch outputs are interfaced to the microcontroller as digital inputs.

Each shoe is also provided with four membrane switches attached at the bottom side of the shoe at pre-defined locations. The locations considered are on the heel part, on the middle lateral side, on the metatarsal area and the last on the big toe. These positions are very important in defining the different phases of gait such as: initial contact, loading response, mid-stance, terminal stance, pre-swing, initial swing, mid-swing and terminal swing. The switch outputs are interfaced to the microcontroller as digital inputs. 
The microcontroller is programed to read the six acceleration signals $(\mathrm{X}, \mathrm{Y}$ and $\mathrm{Z}$ axis accelerations for each foot) and eight digital outputs from switches (four for each foot) for every 5 msecs. The acquired data is immediately transferred to $\mathrm{PC}$ using $\mathrm{X}$-Bee module interfaced to the microcontroller. At the $\mathrm{PC}$, the signals are received by another
X-Bee module, which is directly interfaced to the PC. In PC, the values are received and stored for subsequent analysis, to obtain the different parameters. The X-bee module is programed to have maximum transfer rate of 1, 15,200 baud, to avoid missing of samples.

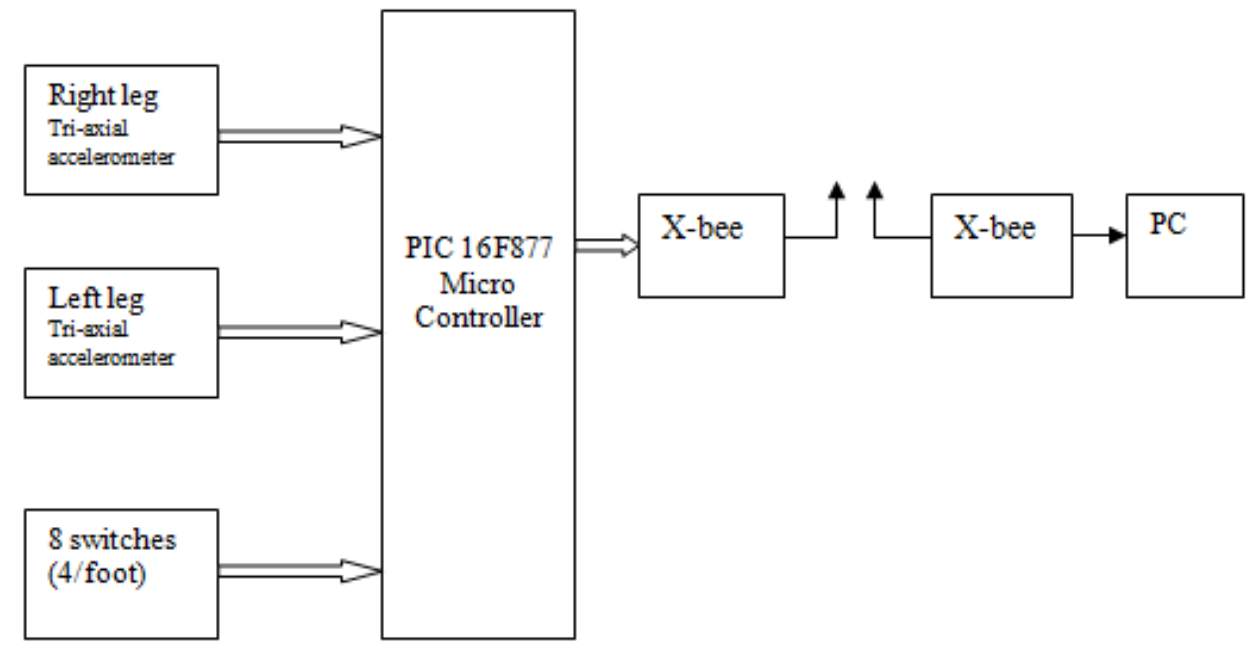

A)Acquisition system

B) Receiver system

Fig. 1. Block diagram of the system

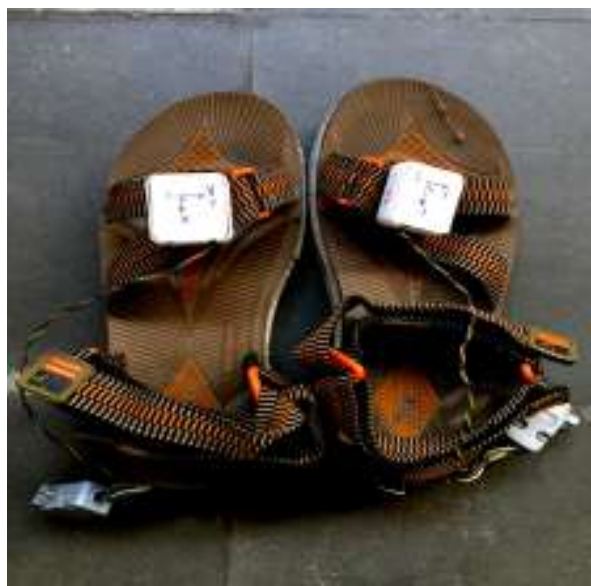

Fig. 2. Shoes with tri-axial accelerometers, 8 membrane switches, X-bee modules located inside the sole

The developed MATLAB software is responsible for reading the values from the com-port (RS-232). The program reads the values from the com-port into an array. Each set of sample consists of 7 values, 6 values corresponding to the two 3 -axis accelerometers mounted on both the foot and last value corresponding to 8 switches (4 switches/ shoe sole). After reading 60 secs data, the MATLAB program closes the comport. A separate program is written for the analysis of the raw signals to obtain different gait parameters. The algorithm initially separates the values pertaining into 3 separate sets acceleration values of both the legs in $\mathrm{x}, \mathrm{y}, \mathrm{z}$ directions. These signals are filtered to remove high frequency noise. The errorfree acceleration signals are then integrated twice to obtain the position signal. To avoid cumulative error due to offset, the output of the second integrator is made zero at the heel switch closure. The parameters obtained from this process are stride lengths, maximum foot rise. Similarly, by using the switch outputs, different time intervals are obtained. The parameters that can be obtained are:

$\mathrm{T} 1=$ Duration of swing phase left leg

$\mathrm{T} 2=$ Duration of swing phase right leg

T3 = Duration of gait cycle L-to-R

T4 = Duration of gait cycle R-to-L

T5 = Duration of single support L-to-R

T6 = Duration of single support R-to-L

$\mathrm{T} 7$ = Duration of double support L-to-R

T8 =Duration of double support R-to-L

\section{3. RESULTS AND DISCUSSION}

In the study, a total of 25 cases have been studied out of which 10 are male and 15 are female, falling under control group. Each subject is explained about the procedure and their written consent is taken. Then each subject is asked to wear the shoe and walk at his/her normal speed. The data is monitored and after getting satisfied, the MATLAB program is allowed to acquire the data for about 60 secs and is stored in PC for subsequent analysis.

The acquired samples are used for extraction of the parameters. To reduce the manual and other errors, ten gait cycles are considered for analysis. From each cycle the gait parameters are obtained and are tabulated for 10 cycles, and then the mean and standard deviation (S.D) are calculated for each parameter separately. The results were tabulated for each parameter for males and females separately. The mean and standard deviation of all gait parameters for males and females are shown in tables 1 and 2 .

From the tables, it can be inferred that, the females normally take quick steps, but with small stride length and the 
foot rise. The percentage of swing phase in total gait cycle is on a average $25-30 \%$ for both males and females. The percentage single support time in total gait cycle is around 5-
$7 \%$. The percentage of double support in total gait cycle is around $12-15 \%$.

TABLE I: GAIT PARAMETERS OF 10 MALE SUBJECTS AND THEIR MEAN AND S.D

\begin{tabular}{|c|c|c|c|c|c|c|c|c|c|c|}
\hline \multirow{2}{*}{ S.No } & T1 msec & $\mathrm{T} 2 \mathrm{msec}$ & T3 msec & $\mathrm{T} 4 \mathrm{~m}$ sec & T5 msec & $\mathrm{T} 6 \mathrm{~m} \mathrm{sec}$ & T7 msec & T8 msec & $\begin{array}{c}\text { Stride } \\
\text { length(c } \\
\text { ms) }\end{array}$ & $\begin{array}{c}\text { Maximum } \\
\text { foot } \\
\text { rise }(\mathrm{cms})\end{array}$ \\
\hline & Mean+S.D & Mean+S.D & Mean+S.D & Mean+S.D & Mean+S.D & Mean+S.D & Mean+S.D & Mean_S.D & $\begin{array}{c}\text { Mean } \pm \\
\text { S.D }\end{array}$ & \begin{tabular}{|l|} 
Mean \pm \\
S.D
\end{tabular} \\
\hline 1 & $530 \pm 11$ & $510 \pm 5$ & $1200 \pm 12$ & $1210 \pm 10$ & $70 \pm 11$ & $80 \pm 5$ & $70 \pm 11$ & $80 \pm 5$ & $30.5 \pm 2.0$ & $18.1 \pm 1.2$ \\
\hline 2 & $460 \pm 11$ & $450 \pm 9$ & $1090 \pm 13$ & $1080 \pm 14$ & $90 \pm 13$ & $90 \pm 12$ & $90 \pm 13$ & $90 \pm 12$ & $27.1 \pm 1.8$ & $21 \pm 1.8$ \\
\hline 3 & $440 \pm 13$ & $440 \pm 14$ & $1080 \pm 19$ & $1090 \pm 14$ & $110 \pm 11$ & $110 \pm 15$ & $110 \pm 11$ & $110 \pm 15$ & $29.3 \pm 1.4$ & $16 \pm 0.9$ \\
\hline 4 & $500 \pm 11$ & $460 \pm 9$ & $1090 \pm 7$ & $1090 \pm 12$ & $80 \pm 8$ & $60 \pm 5$ & $80 \pm 8$ & $60 \pm 5$ & $35.2 \pm 2.2$ & $19.3 \pm 1.2$ \\
\hline 5 & $470 \pm 16$ & $450 \pm 8$ & $1030 \pm 20$ & $1030 \pm 21$ & $60 \pm 13$ & $50 \pm 6$ & $60 \pm 13$ & $50 \pm 6$ & $31.2 \pm 1.5$ & $20.2 \pm 1.6$ \\
\hline 6 & $470 \pm 6$ & $460 \pm 10$ & $1030 \pm 10$ & $1030 \pm 11$ & $60 \pm 5$ & $50 \pm 4$ & $60 \pm 5$ & $50 \pm 4$ & $28.7 \pm 2.5$ & $18.1 \pm 1.3$ \\
\hline 7 & $460 \pm 12$ & $450 \pm 9$ & $1010 \pm 15$ & $1010 \pm 15$ & $60 \pm 8$ & $40 \pm 5$ & $60 \pm 8$ & $40 \pm 5$ & $25.6 \pm 2.0$ & $21.5 \pm 1.2$ \\
\hline 8 & $500 \pm 11$ & $440 \pm 11$ & $1040 \pm 12$ & $1030 \pm 12$ & $60 \pm 13$ & $40 \pm 4$ & $60 \pm 13$ & $40 \pm 4$ & $29.1 \pm 1.8$ & $19.7 \pm 1.3$ \\
\hline 9 & $490 \pm 10$ & $450 \pm 24$ & $1060 \pm 16$ & $1060 \pm 14$ & $60 \pm 13$ & $50 \pm 5$ & $60 \pm 13$ & $50 \pm 5$ & $28.3 \pm 1.7$ & $20.2 \pm 1.1$ \\
\hline 10 & $430 \pm 8$ & $460 \pm 22$ & $1050 \pm 12$ & $1060 \pm 7$ & $60 \pm 8$ & $70 \pm 10$ & $60 \pm 8$ & $70 \pm 10$ & $32.1 \pm 2.1$ & $19.8 \pm 1.5$ \\
\hline $\begin{array}{l}\text { Meant } \\
\text { S.D }\end{array}$ & $475 \pm 30$ & $475 \pm 20$ & $1068 \pm 54$ & $1069 \pm 51$ & $71 \pm 17$ & $64 \pm 23$ & $71 \pm 17$ & $64 \pm 23$ & $30 \pm 3$ & $19 \pm 2$ \\
\hline
\end{tabular}

TABLE II GAIT PARAMETERS OF 15 FEMALE SUBJECTS AND THEIR MEAN AND S.D

\begin{tabular}{|c|c|c|c|c|c|c|c|c|c|c|}
\hline S.No & $\mathrm{T} 1 \mathrm{msec}$ & $\mathrm{T} 2 \mathrm{msec}$ & $\mathrm{T} 3 \mathrm{msec}$ & $\mathrm{T} 4 \mathrm{msec}$ & T5 msec & T6 msec & $\begin{array}{c}\mathrm{T} 7 \\
\mathrm{msec}\end{array}$ & $\begin{array}{c}\text { T8 } \\
\text { msec }\end{array}$ & $\begin{array}{c}\text { Stride } \\
\text { length }(\mathrm{cm} \\
\mathrm{s})\end{array}$ & $\begin{array}{l}\text { Maximum } \\
\text { foot rise } \\
\text { (cms) }\end{array}$ \\
\hline & $\begin{array}{c}\text { Mean } \pm \\
\text { S.D }\end{array}$ & Mean+S.D & Mean+S.D & Mean+S.D & Mean+S.D & Mean \pm S.D & $\underset{\text { S.D }}{\text { Mean } \pm}$ & $\underset{\text { S.D }}{\text { Mean } \pm}$ & Mean+S.D & Mean+S.D \\
\hline 1 & $470 \pm 14$ & $470 \pm 7$ & $1050 \pm 19$ & $1050 \pm 21$ & $570 \pm 25$ & $580 \pm 21$ & $60 \pm 7$ & $60 \pm 18$ & $27.3 \pm 2.3$ & $13.2 \pm 1.2$ \\
\hline 2 & $440 \pm 7$ & $620 \pm 16$ & $1050 \pm 13$ & $1060 \pm 19$ & $610 \pm 11$ & $440 \pm 28$ & $90 \pm 5$ & $90 \pm 10$ & $23.1 \pm 1.9$ & $12.6 \pm 0.7$ \\
\hline 3 & $470+9$ & $460 \pm 15$ & $1080 \pm 10$ & $1070 \pm 14$ & $610 \pm 16$ & $610 \pm 19$ & $80 \pm 5$ & $70 \pm 8$ & $29.2 \pm 1.5$ & $14.2 \pm 1.3$ \\
\hline 4 & $430 \pm 8$ & $430 \pm 18$ & $1010 \pm 6$ & $1020 \pm 8$ & $580 \pm 12$ & $590 \pm 14$ & $80 \pm 12$ & $80 \pm 7$ & $24.2 \pm 2.1$ & $10.3 \pm 1.7$ \\
\hline 5 & $480 \pm 9$ & $470 \pm 7$ & $1060 \pm 12$ & $1060 \pm 8$ & $580 \pm 10$ & $590 \pm 11$ & $70 \pm 13$ & $60 \pm 8$ & $28.2 \pm 1.9$ & $12.5 \pm 1.1$ \\
\hline 6 & $430 \pm 7$ & $430 \pm 15$ & $1010 \pm 13$ & $1020 \pm 11$ & $580 \pm 14$ & $590 \pm 15$ & $70 \pm 15$ & $60 \pm 13$ & $27.0 \pm 2.1$ & $11.8 \pm 1.5$ \\
\hline 7 & $470 \pm 10$ & $610 \pm 23$ & $1060 \pm 22$ & $1070 \pm 10$ & $590 \pm 13$ & $460 \pm 19$ & $70 \pm 12$ & $80 \pm 5$ & $28.5 \pm 1.7$ & $12.9 \pm 0.9$ \\
\hline 8 & $470 \pm 8$ & $430 \pm 14$ & $1040 \pm 19$ & $1050 \pm 13$ & $570 \pm 21$ & $620 \pm 21$ & $70+9$ & $80 \pm 10$ & $27.2+1.9$ & $13.6 \pm 1.5$ \\
\hline 9 & $520 \pm 5$ & $490 \pm 19$ & $1130 \pm 24$ & $1140 \pm 18$ & $620 \pm 21$ & $640 \pm 12$ & $60 \pm 3$ & $70 \pm 8$ & $31.1 \pm .5$ & $14.2 \pm 1.7$ \\
\hline 10 & $470 \pm 8$ & $430 \pm 14$ & $1040 \pm 19$ & $1050 \pm 13$ & $570 \pm 21$ & $620 \pm 21$ & $70 \pm 11$ & $80 \pm 10$ & $29.5 \pm 1.2$ & $13.3 \pm 0.9$ \\
\hline 11 & $480 \pm 12$ & $600 \pm 12$ & $1040 \pm 15$ & $1040 \pm 10$ & $560 \pm 13$ & $600 \pm 12$ & $70 \pm 17$ & $50 \pm 10$ & $26.5 \pm 1.1$ & $12.9 \pm 1.5$ \\
\hline 12 & $450 \pm 8$ & $450 \pm 23$ & $1050 \pm 14$ & $1060 \pm 15$ & $600 \pm 15$ & $610 \pm 22$ & $110 \pm 7$ & $70 \pm 10$ & $25.0 \pm 1.7$ & $11.9 \pm 1.2$ \\
\hline 13 & $520 \pm 12$ & $470 \pm 10$ & $1100 \pm 15$ & $1100 \pm 12$ & $590 \pm 10$ & $620 \pm 10$ & $70 \pm 5$ & $40 \pm 12$ & $27.2 \pm 0.9$ & $14.1 \pm .5$ \\
\hline 14 & $440 \pm 15$ & $430 \pm 21$ & $1010 \pm 15$ & $1020 \pm 11$ & $580 \pm 12$ & $590 \pm 23$ & $70 \pm 12$ & $80 \pm 11$ & $24.5 \pm 1.2$ & $12.7 \pm 0.9$ \\
\hline 15 & $470 \pm 9$ & $460 \pm 15$ & $1080 \pm 10$ & $1070 \pm 14$ & $610 \pm 16$ & $610 \pm 19$ & $80 \pm 5$ & $70 \pm 8$ & $24.9+1.2$ & $12.1 \pm 1.9$ \\
\hline $\begin{array}{l}\text { Mea } \\
\mathrm{n} \pm \\
\text { S.D }\end{array}$ & $467 \pm 27$ & $483 \pm 68$ & $1054 \pm 34$ & $1059 \pm 31$ & $588 \pm 18$ & $585 \pm 57$ & $75 \pm 12$ & $09 \pm 13$ & $27 \pm 2$ & $13 \pm 1$ \\
\hline
\end{tabular}




\section{CONCLUSION AND FUTURE SCOPE}

In the present project, a wearable sensor based cost effective solution is provided for the determination of various gait parameters. Here, only control group is considered, but it can be extended by considering different pathological cases and age related issues. Different algorithms can be identified to differential normal and various pathological cases and gait abnormalities due to age. This can be further extended to provide guidance to the physician to make corrective steps in treatment process.

\section{REFERENCES}

[1] I.A. Karaulovaa,*, P.M. Hall, A.D. Marshall, "Tracking people in three dimensions using a hierarchical model", Elsevier Science, Image and Vision Computing 20 (2002) 691-700.

https://doi.org/10.1016/S0262-8856(02)00059-8

[2] Aurelio Cappozzo, "Gait analysis methodology" Human Movement Science 3(1-2):27-50 • March 1984

[3] Maluf KS, Morley RE Jr, Richter EJ, Klaesner JW, Mueller MJ, "Monitoring in-shoe plantar pressures, temperature, and humidity: reliability and validity of measures from a portable device", Arch Phys Med Rehabil. 2001 Aug;82(8):1119-27. https://doi.org/10.1053/apmr.2001.24223

[4] I.P PAPPAS; T.KelleR; S.MANGOLD; M.R.POPOVIC; V.DIETZ; M.MORARI, "A RELIABLE GYROSCOPE-BASED GAIT-PHASE DETECTION SENSOR EMBEDDED IN A SHOE INSOLE", IEEE SENSORS JOURNAL ( VOLUME: 4, ISSUE: 2, APRIL 2004 )

[5] Mathie MJ, Coster AC, Lovell NH, Celler BG, "Accelerometry: providing an integrated, practical method for long-term, ambulatory monitoring of human movement", J Biomech, Physiol Meas. 2004 Apr;25(2):R1-20.

https://doi.org/10.1088/0967-3334/25/2/R01

[6] SimON SR, "QUANTIFICATION OF HUMAN MOTION: GAIT ANALYSISBENEFITS AND LIMITATIONS TO ITS APPLICATION TO CLINICAL PROBLEMS", 2004 DEC;37(12):1869-80. 